\title{
On the Poisson Structure of the Time-Dependent Mean-Field Equations For Systems of Bosons out of Equilibrium
}

\author{
Mohamed Benarous * \\ Division de Physique Théorique ! \\ Institut de Physique Nucléaire, \\ F-91406, Orsay Cedex, France
}

September 26, 2017

\begin{abstract}
We analyze the Poisson structure of the time-dependent mean-field equations for bosons and construct the Lie-Poisson bracket associated to these equations. The latter follow from the time-dependent variational principle of Balian and Vénéroni when a gaussian Ansatz is chosen for the density operator. We perform a stability analysis of
\end{abstract}

*Perm. address: University of Chlef, BP 151, 02000 Chlef, Algeria.

${ }^{\dagger}$ Unité de Recherche des Universités Paris XI et Paris VI associée au C.N.R.S. 
both the full and the linearized equations. We also search for the canonically conjugate variables. In certain cases, the evolution equations can indeed be cast in a Hamiltonian form.

PACS numbers : 05.30.-d, 05.30.Jp, 03.65.-w, 03.65.Fd 


\section{Introduction}

Poisson structures have already been used to study various properties of classical theories such as the Maxwell-Vlasov equations and the BBGKY hierarchy [5, 6]. They are useful in quantization methods; for a review, see [2, 7, 8]. In the quantum case, besides the abstract quantum group approach, Poisson structures have found some applications in the manybody problem. In particular, they have been used to study the stability properties of important types of approximation such as the static HartreeFock $(\mathrm{HF})$, the time-dependent Hartree-Fock (TDHF) and the random phase approximation (RPA), both in the zero temperature [1, 9] and in the thermal case [四]. With their help, the connections between these theories, as well as with classical dynamics, follow in a very transparent fashion.

The purpose of the present paper is to extend the analysis of ref.[四, which deals with fermions, to the time evolution of many-boson systems. Among the relevant problems that we have in mind, we can quote bose condensation phenomenon[10, 11, 12]. In particular, recent experiments on the Bose-Einstein condensation of dilute atomic gases in laser traps at ultracold temperatures [13] are raising a wide interest. They involve not only the study of equilibrium properties, but also that of dynamics. In the existing mean-field approaches, this dynamics deals with the expectation value of a creation or an annihilation operator, which is the order parameter, and more generally with the expectation values of product of two such operators. Our purpose is to analyze the structure of these equations so as to clarify their meaning. 
Our paper is organized as follows. We briefly present in section 2.1 the time-dependent variational principle proposed by Balian and Vénéroni (BV) [14, 16] which we use to derive the approximate evolution equations. We also introduce, as well as some useful notations, the trial subspaces which we will consider throughout this work. These ansätze lead to the Time-Dependent Hartree-Bogoliubov (TDHB) equations. They belong to the class of the time-dependent mean-field approximations and they are the bosonic counterpart of the time-dependent Hartree-Fock (TDHF) equations for fermions. In section 3, we construct the Lie-Poisson bracket associated with these TDHB equations. This is achieved by extending the analysis of ref. [4] to bose systems. Our formultation includes the possible existence of bose condensates. We derive in section 4 the corresponding linearized equations and study their stability in the sense of Lyapunov. A question that arises naturally in this context is whether canonically conjugate variables can be found. We perform a canonical transformation allowing us to rewrite the TDHB equations in a form which, in some circumstances, is well suited to the discussion of this question. The last section contains some conclusions.

\section{The Bosonic TDHB Equations}

\subsection{The time-dependent variational principle}

The BV variational principle [14, 16] is devised to evaluate the expectation value $\operatorname{Tr} A D\left(t_{1}\right)$ of a given observable $A$, measured at a final time $t_{1}$, when the density operator $D_{0}$ is known at the initial time $t_{0}$. To this aim, one 
introduces the action-like functional

$$
\mathcal{I}\{\mathcal{A}(t), \mathcal{D}(t)\} \equiv \operatorname{Tr} \mathcal{A}\left(t_{0}\right) D\left(t_{0}\right)+\int_{t_{0}}^{t_{1}} \mathrm{~d} t \operatorname{Tr} \mathcal{D}(t)\left(\frac{\mathrm{d} \mathcal{A}(t)}{\mathrm{d} t}-i[\mathcal{A}(t), H]\right)
$$

where the variational quantities are the time-dependent operators $\mathcal{D}(t)$ and $\mathcal{A}(t)$, the latter being subject to the final boundary condition

$$
\mathcal{A}\left(t_{1}\right)=A
$$

The symbol Tr stands for a trace over the complete Fock space (we take $\hbar=1)$. The desired quantity is then provided by the stationary value of the functional (2.1), where $H$ is the Hamiltonian of the system.

When the allowed variations $\delta \mathcal{A}(t)$ and $\delta \mathcal{D}(t)$ are unrestricted, the stationarity conditions of the BV functional (2.1) lead to the exact Liouvillevon-Neumann equation for $\mathcal{D}(t)$ and to the (backward) Heisenberg equation for $\mathcal{A}(t)$. When restricted trial ansätze are chosen for $\mathcal{A}(t)$ and $\mathcal{D}(t)$, the extremization of (2.1) yields approximate, and in general coupled, evolution equations for $\mathcal{A}(t)$ and $\mathcal{D}(t)$.

\subsection{Trial spaces and the TDHB equations}

Let us first introduce the trial class of operators, which we shall use throughout this work for $\mathcal{D}(t)$ and $\mathcal{A}(t)$. To this end, we define the $2 n$ component operator $\alpha$

$$
\alpha=\left(\begin{array}{l}
a \\
a^{\dagger}
\end{array}\right),
$$

where $a^{\dagger}$ and $a$ denote boson creation and annihilation operators in a given single-particle space of dimension $n$. In terms of the $\alpha$ 's, the usual commu- 
tation relations can be expressed in the compact form $(i, j=1 \ldots 2 n)$

$$
\left[\alpha_{i}, \alpha_{j}\right]=\tau_{i j} \quad, \quad \tau=i \sigma_{2}=\left(\begin{array}{rr}
0 & 1_{n} \\
-1_{n} & 0
\end{array}\right),
$$

where $1_{n}$ is the $(n \times n)$ unit matrix and $\sigma_{2}$ the $(2 n \times 2 n)$ second Pauli matrix.

With this notation, our trial class for the operator $\mathcal{D}(t)$, the exponentials of the linear plus quadratic forms in $\alpha$, can be written in the factorized form [17, 19]

$$
\mathcal{D}=\exp (\nu) \exp (\tilde{\lambda} \tau \alpha) \exp \left(\frac{1}{2} \tilde{\alpha} \tau S \alpha\right)
$$

where $\nu$ is a c-number, $\lambda$ a $2 n$-component vector and $S$ a $(2 n \times 2 n)$ symplectic matrix ( $\tau S$ is symmetric). The tilda symbol denotes vector or matrix transposition. For any operator $O$, let us denote the average value $\langle O\rangle$ and the shifted operator $\bar{O}$ by $\langle O\rangle=\operatorname{Tr} O \mathcal{D} / \mathcal{Z}$ and $\bar{O}=O-\langle O\rangle$. Then, $\mathcal{D}$ is completely specified by the knowledge of "the partition function" $\mathcal{Z} \equiv \operatorname{Tr} \mathcal{D}$, the vector $\langle\alpha\rangle$ and the contraction matrix $\rho$ (which we shall also call the single-particle density matrix) defined as

$$
\rho_{i j} \equiv\left\langle(\tau \bar{\alpha})_{j} \bar{\alpha}_{i}\right\rangle
$$

For our purpose, the parameterization of the operator $\mathcal{D}$ by $\mathcal{Z},\langle\alpha\rangle$ and $\rho$ is more convenient than that by $\nu, \lambda$ and $S$. In particular, we will see that the evolution equations for the former variables take a simple form.

With the same notations, we take as our variational choice [17, 18] for the operator $\mathcal{A}(t)$ a linear plus quadratic form in the operator $\alpha$ :

$$
\mathcal{A}(t)=\nu_{a}(t)+\tilde{\lambda}_{a}(t) \tau \alpha+\frac{1}{2} \tilde{\alpha} \tau S_{a}(t) \alpha
$$


to which we shall loosely refer as a single-particle operator.

With the choices (2.5) and (2.7), it is possible to write explicitly the functional (2.1) in terms of the variational parameters $\mathcal{Z},\langle\alpha\rangle, \rho, \nu_{a}, \lambda_{a}$ and $S_{a}$ [17]. Indeed, we find

$$
\begin{aligned}
\mathcal{I}= & \operatorname{Tr} A \mathcal{D}\left(t_{1}\right)-\int_{t_{0}}^{t_{1}} \mathrm{~d} t \\
& \left(\operatorname{Tr} \mathcal{A} \mathcal{D} \frac{\dot{\mathcal{Z}}}{\mathcal{Z}}-i \mathcal{Z}\left\{\tilde{L}_{a} \tau\left(i\langle\dot{\alpha}\rangle-\tau \frac{\partial \mathcal{E}}{\partial\langle\alpha\rangle}\right)-\frac{1}{2} \operatorname{tr} S_{a}\left(i \dot{\rho}+2\left[\rho, \frac{\partial \mathcal{E}}{\partial \rho}\right]\right)\right\}\right),
\end{aligned}
$$

with $L_{a}=\lambda_{a}-S_{a}\langle\alpha\rangle$. The TDHB equations

$$
\left\{\begin{aligned}
i \dot{\mathcal{Z}} & =0, \\
i\langle\dot{\alpha}\rangle & =\tau \frac{\partial \mathcal{E}}{\partial\langle\alpha\rangle}, \\
i \dot{\rho} & =-2\left[\rho, \frac{\partial \mathcal{E}}{\partial \rho}\right],
\end{aligned}\right.
$$

are then obtained [17, 18] as the stationarity conditions of the reduced form (2.8) of the BV functional (2.1) with respect to the parameters $\nu_{a}, \lambda_{a}$ and $S_{a}$. In these equations, $\mathcal{E} \equiv\langle H\rangle$ is the mean-field energy and the dots denote $\frac{\mathrm{d}}{\mathrm{d} t}$. We have used the usual convention $(\partial / \partial \rho)_{i j}=\partial / \partial \rho_{j i}$.

Thus, within the framework of the BV variational principle (2.1), and within the trial class (2.5) for the density operator, the time-dependent meanfield equations (2.9) appear to optimize the expectation values of singleparticle observables.

The mean-field energy is a sum of contributions of the type $\rho$ and $\langle\alpha\rangle\langle\alpha\rangle$ for its kinetic part, $\rho \rho, \rho\langle\alpha\rangle\langle\alpha\rangle$ and $\langle\alpha\rangle\langle\alpha\rangle\langle\alpha\rangle\langle\alpha\rangle$ for its potential part. Hence the equations (2.9) couple the dynamical variables $\langle\alpha\rangle$ and $\rho$. If this coupling is disregarded, and if the interaction has zero range, the equation for $\langle\alpha\rangle$ reduces to the Gross-Pitaevskii equation [12]. 
The equations (2.9) do not depend on the parameters $\nu_{a}(t), \lambda_{a}(t)$ and $S_{a}(t)$ : the evolution of the operator $\mathcal{D}(t)$ decouples from the evolution of $\mathcal{A}(t)$. The knowledge of $\nu_{a}(t), \lambda_{a}(t)$ and $S_{a}(t)$ is unnecessary for the determination of $\mathcal{D}(t)$, and therefore of $\operatorname{Tr} A \mathcal{D}\left(t_{1}\right)$. The solution of eqs.(2.9) is uniquely defined once the initial conditions $\mathcal{Z}\left(t_{0}\right),\langle\alpha\rangle\left(t_{0}\right)$ and $\rho\left(t_{0}\right)$ are specified. It should be noticed that, in the present context, this feature results from the linearity in the variational parameters of the Ansatz (2.7). Otherwise, in the case of a non-linear parametrization, one should have to consider simultaneously the evolution equations for $\mathcal{A}(t)$ obtained by writing the stationarity conditions of (2.1) with respect to the parameters characterizing $\mathcal{D}(t)$. Then, the motions of $\mathcal{D}(t)$ and $\mathcal{A}(t)$ would be coupled; moreover, the boundary conditions on $\mathcal{D}\left(t_{0}\right)$ and $\mathcal{A}\left(t_{1}\right)$ would lead to a mixed boundary value problem 14, 18].

Although we shall not discuss them in the present paper, the equations of motion of $\mathcal{A}(t)$ have their own interest; indeed, they are tightly related to the random phase approximation (RPA) and they play a crucial role 20 for the coherent determination of fluctuations and (two-time) correlation functions of the single-particle observables (2.7).

Let us now discuss briefly some general propreties of the TDHB equations (2.9) (see [15, 17] for a more detailed discussion). We note first that the "partition function" $\mathcal{Z}$ is conserved; this is automatically entailed by the $\mathrm{BV}$ variational principle when the allowed variations $\delta \mathcal{A}(t)$ includes the unit operator. Other properties of the TDHB equations are the conservation of the mean-field energy $\mathcal{E}$ (for a time-independent Hamiltonian) and the unitary evolution of the contraction matrix $\rho(t)$. The latter means that the 
eigenvalues of $\rho(t)$ are conserved. This implies in particular the conservation of the single-particle von-Neumann entropy $\mathcal{S}=-\operatorname{Tr} \mathcal{D} \log \mathcal{D}$ and that an initially pure state $\rho(\rho+1)=0$ remains pure during the TDHB evolution. The conservation of the free energy $\mathcal{F}=\mathcal{E}-\beta^{-1} \mathcal{S}$ naturally follows $\left(\beta^{-1}\right.$ is the temperature.) We will see the interest of this property in the next sections. These conservation laws show that the TDHB approximation reproduces, in the reduced single-particle space, some of the exact ones.

\section{The Lie-Poisson Structure of the TDHB Equations}

We now examine the mathematical structure of the TDHB equations (2.9). A first interest of this analysis is to establish a close connection between the TDHB equations and dynamical systems [3, 21, 22]. It may prove useful in the elaboration of more sophisticated approximations.

It was pointed out in ref. [4] that the TDHF equations possess a LiePoisson structure. The reason is that they deal with the expectation values of the one-body operators $a_{i}^{\dagger} a_{j}$ which form a Lie algebra. In the boson case, the set of the operators $\{1, \alpha, \alpha \alpha\}$ form also a Lie algebra. One expects, therefore, the existence of a Lie-Poisson bracket associated with the TDHB equations (2.9). This existence results also directly from a theorem which can be found in refs. [7, 23]). In order to construct the corresponding Lie-Poisson bracket, let us introduce the set of the relevant operators $b^{\mu}(\mu=1,2,3)$ 
defined as follows:

$$
b^{1}=1 \quad, \quad b_{i}^{2}=\alpha_{i} \quad, \quad b_{i j}^{3}=(\tau \bar{\alpha})_{j} \bar{\alpha}_{i}
$$

These operators satisfy the Lie-algebra

$$
\left[b^{\mu}, b^{\nu}\right]=f_{\sigma}^{\mu \nu} b^{\sigma}
$$

Using the commutation relations (2.4), we find that the nonvanishing structure constants $f_{\sigma}^{\mu \nu}$ are:

$$
\left\{\begin{aligned}
&\left(f_{1}^{22}\right)_{i j}=\tau_{i j} \\
&\left(f_{1}^{23}\right)_{i, j k}=\left(f_{1}^{32}\right)_{j i, k}=\delta_{i k}\langle\alpha\rangle_{j}-\tau_{i j}(\tau\langle\alpha\rangle)_{k}, \\
&\left(f_{2}^{23}\right)_{i, j k}^{l}=\left(f_{2}^{32}\right)_{j i, l}^{k}=-\delta_{i k} \delta_{j l}+\tau_{i j} \tau_{k l} \\
&\left(f_{3}^{33}\right)_{i j, k l}^{m n}=\delta_{i l} \delta_{j m} \delta_{k n}-\delta_{j k} \delta_{l m} \delta_{i n}+\tau_{i k} \delta_{j m} \tau_{l n}-\tau_{j l} \delta_{i n} \tau_{k m}
\end{aligned}\right.
$$

The Lie-Poisson bracket associated with the TDHB equations can now be constructed as follows. Consider the quantities $z^{\mu}=\left\langle b^{\mu}\right\rangle$ which are directly related to our dynamical variables $\mathcal{Z},\langle\alpha\rangle$ and $\rho$. The quantity $z=\left(z^{1}, z^{2}, z^{3}\right)$ is a vector in the Liouville space. This suggests defining the Poisson tensor $\left\{z^{\mu}, z^{\nu}\right\}$ as

$$
\mathcal{C}^{\mu \nu} \equiv\left\{z^{\mu}, z^{\nu}\right\}=f_{\sigma}^{\mu \nu} z^{\sigma}
$$

Upon using (3.3), we can see that $\mathcal{C}^{\mu \nu}$ has only two non-zero components, namely

$$
\left\{\begin{aligned}
\left(\mathcal{C}^{22}\right)_{i j} & =\tau_{i j}, \\
\left(\mathcal{C}^{33}\right)_{i j, k l} & =\delta_{i l} \rho_{k j}-\delta_{k j} \rho_{i l}+\tau_{i k}(\tau \rho)_{l j}-\tau_{l j}(\rho \tau)_{i k} .
\end{aligned}\right.
$$

Obviously, $\mathcal{C}^{\mu \nu}$ is antisymmetric with respect to its lower indices owing to the antisymmetry of the matrix $\tau$ and to the property $\rho_{j i}=(\tau(1+\rho) \tau)_{i j}$ (see 2.6). 
We define the Lie-Poisson bracket of two functions $h(z)$ and $g(z)$, depending on the components of the variables $z^{\mu}$ ), in the usual way [7]:

$$
\{h, g\}=\frac{1}{i} \frac{\partial h}{\partial z^{\mu}} \mathcal{C}^{\mu \nu} \frac{\partial g}{\partial z^{\nu}} .
$$

This expression is clearly bilinear and antisymmetric. In addition, it satisfies Leibniz's derivation rule

$$
\{h g, k\}=h\{g, k\}+\{h, k\} g,
$$

and Jacobi's identity. This last property follows directly from the Lie algebra (3.2). Replacing in (3.6) the function $h$ by one component of the variables $z$ and using (3.5), we obtain for any function $g$

$$
\left\{\begin{aligned}
\{1, g\} & =0 \\
\left\{\langle\alpha\rangle_{i}, g\right\} & =\frac{1}{i}\left(\tau \frac{\partial g}{\partial\langle\alpha\rangle}\right)_{i} \\
\left\{\rho_{i j}, g\right\} & =-\frac{2}{i}\left[\rho, \frac{\partial g}{\partial \rho}\right]_{i j}
\end{aligned}\right.
$$

In particular, when $g$ is the energy or the free energy, we can express the TDHB equations in the form

$$
\dot{z}^{\mu}=\left\{z^{\mu}, \mathcal{E}(z)\right\}=\left\{z^{\mu}, \mathcal{F}(z)\right\}
$$

which can also be written as

$$
\dot{z}^{\mu}=\frac{1}{i} \mathcal{C}^{\mu \nu} \frac{\partial \mathcal{F}}{\partial z^{\nu}}
$$

The expressions (3.9-3.10) clearly exhibit the classical structure of the TDHB equations, with the mean-field energy $\mathcal{E}$, or the free energy $\mathcal{F}$, playing the role of an Hamiltonian. However, the non-symplectic Poisson tensor (3.5) induces a non-canonical hamiltonian structure (also called a Lie-Poisson system). 
From (3.10), one sees that the evolution of any differentiable function $h(z)$ is governed by the equation

$$
\frac{\mathrm{d} h(z)}{\mathrm{d} t}=\{h(z), \mathcal{F}(z)\} .
$$

In particular, any function $h(z)$ which "Poisson-commutes" with the free energy (or the energy) is a constant of the TDHB motion.

\section{Linearized Equations and Stability Analysis}

Let $z_{0}$ be a static solution of the TDHB equations (3.9):

$$
\left\{z_{0}^{\mu}, \mathcal{F}\left(z_{0}\right)\right\}=\frac{1}{i} \mathcal{C}^{\mu \nu}\left(z_{0}\right) \frac{\partial \mathcal{F}}{\partial z_{0}^{\nu}}=0 .
$$

We consider a small amplitude motion $\delta z$ around $z_{0}$ such that $z^{\mu}=z_{0}^{\mu}+\delta z^{\mu}$. Using (3.9) and retaining only linear terms in $\delta z$, one obtains

$$
\frac{\mathrm{d}}{\mathrm{d} t}\left(\delta z^{\mu}\right)=\frac{1}{i} \mathcal{C}^{\mu \nu}\left(z_{0}\right) \frac{\partial \mathcal{F}_{2}}{\partial\left(\delta z^{\nu}\right)},
$$

where $\mathcal{F}_{2}$ is the expansion up to second order of the free energy $\mathcal{F}$ :

$$
\mathcal{F}_{2}(z)=\mathcal{F}\left(z_{0}\right)+\frac{\partial \mathcal{F}}{\partial z_{0}^{\mu}} \delta z^{\mu}+\frac{1}{2} \frac{\partial^{2} \mathcal{F}}{\partial z_{0}^{\mu} \partial z_{0}^{\nu}} \delta z^{\nu} \delta z^{\mu} .
$$

The linearized TDHB equations (LTDHB) can therefore be written in the form

$$
\frac{\mathrm{d}}{\mathrm{d} t}\left(\delta z^{\mu}\right)=\left\{\delta z^{\mu}, \mathcal{F}_{2}(z)\right\}_{0}
$$


the Lie-Poisson bracket $\{,\}_{0}$ being given by (3.6) with the Poisson tensor evaluated at the point $z_{0}$. The LTDHB equations also exhibit a classical structure with $\mathcal{F}_{2}$ playing the role of an Hamiltonian (which we note is a constant of the LTDHB motion) and with the non-symplectic Poisson tensor $\mathcal{C}^{\mu \nu}\left(z_{0}\right)$.

The stability analysis of TDHB and LTDHB relies on the LagrangeDirichlet theorem (ref.[四] sect. 3.2). The TDHB motion is Lyapunov stable (that is, a trajectory starting in the neighborhood of $z_{0}$ remains in this neighborhood during the evolution) if $z_{0}$ is a local minimum of $\mathcal{F}$. The same condition (with $\mathcal{F}$ replaced by $\mathcal{F}_{2}$ ) ensures the Lyapunov stability of the

LTDHB motion provided the stability matrix $\mathcal{B}_{\mu \nu}=\left.\frac{\partial^{2} \mathcal{F}}{\partial z^{\mu} \partial z^{\nu}}\right|_{z=z_{0}}$ has only positive eigenvalues. In this case, the TDHB equations are said to be linearly stable. One notes that Lyapunov stability and linear stability are independent of each other since the non-linear terms in the TDHB equations may have stabilizing or destabilizing effects on the dynamics.

Let us finally mention that the previous results hold both for pure states and at non-zero temperature. In the first case, one should evidently replace the Helmholtz free energy $\mathcal{F}$ by the mean-field energy $\mathcal{E}$.

\section{$5 \quad$ Search for Conjugate Variables}

We have seen in sections 3 and 4 that both TDHB and LTDHB admit a non-canonical hamiltonian structure. One may therefore wonder about the existence of canonically conjugate variables (CCV). This analysis is of course of great interest in the study of the classical limit or in the "re-quantization" 
process of the mean-field approximation.

In principle, the CCV are found by diagonalizing the Poisson tensor (3.4). The second block $\mathcal{C}^{22}$ being $z$-independent (see 3.5), one can easily find a global canonical parametrization for $z^{2}$. Indeed, by using the usual canonical transformations (where $j=1 \ldots n$ )

$$
a_{j}=\frac{1}{\sqrt{2}}\left(x_{j}+i p_{j}\right) \quad, \quad a_{j}^{\dagger}=\frac{1}{\sqrt{2}}\left(x_{j}-i p_{j}\right),
$$

(which underlie the equivalence between the algebra of boson operators and the symplectic algebra of canonical pairs of the position and momentum operators $x$ and $p$ ), and upon defining the variables

$$
\left\{\begin{array}{l}
\varphi_{i}=\left\langle x_{i}\right\rangle, \\
\pi_{i}=\left\langle p_{i}\right\rangle,
\end{array}\right.
$$

the evolution of $\langle\alpha\rangle$ translates into the equations

$$
\left\{\begin{array}{l}
\dot{\varphi}=\frac{\partial \mathcal{F}}{\partial \pi}, \\
\dot{\pi}=-\frac{\partial \mathcal{F}}{\partial \varphi},
\end{array}\right.
$$

which are clearly in an Hamiltonian form, and which, we recall, are coupled to $\rho$ through $\mathcal{F}$.

In order to discuss the possibility of a canonical parametrization of $z^{3}$, one must note that the block $\mathcal{C}^{33}$ depends on the point $z$ (more precisely, on $\rho$ ). One looks therefore for local CCV (there is no rigorous proof of nonexistence, but the search for global sets of CCV seems very hazardous.) Let us consider the instructive (and simpler) example of the LTDHB equations (4.4) in a basis where $\rho_{0}$ is diagonal: $\rho_{0 i j}=\rho_{0 i} \delta_{i j}$. The equation (3.5) gives

$$
\left(\mathcal{C}^{33}\right)_{i j, k l}=\left(\rho_{0 j}-\rho_{0 i}\right)\left(\delta_{i l} \delta_{k j}+\tau_{i k} \tau_{l j}\right),
$$


and the LTDHB equations write

$$
\frac{\mathrm{d}}{\mathrm{d} t} \delta \rho_{i j}=i\left(\rho_{0 i}-\rho_{0 j}\right)\left(\frac{\partial \mathcal{F}_{2}}{\partial(\delta \rho)}+\tau \frac{\partial \mathcal{F}_{2}}{\partial(\delta \tilde{\rho})} \tau\right)_{i j} .
$$

It is easily seen that when $\rho_{0 i}-\rho_{0 j}>0$, one can define the variables

$$
q_{i j}=\frac{\delta \rho_{i j}+\delta \rho_{j i}}{\sqrt{2\left(\rho_{0 i}-\rho_{0 j}\right)}} \quad, \quad p_{i j}=\frac{i\left(\delta \rho_{i j}-\delta \rho_{j i}\right)}{\sqrt{2\left(\rho_{0 i}-\rho_{0 j}\right)}},
$$

of which the evolution takes the form of Hamilton's equations

$$
\dot{q}_{i j}=\frac{\partial \mathcal{F}_{2}}{\partial p_{i j}} \quad, \quad \dot{p}_{i j}=-\frac{\partial \mathcal{F}_{2}}{\partial q_{i j}} .
$$

However, for $\rho_{0 i}=\rho_{0 j}$ (degeneracy in $\rho$ ), we see on (5.5) that one must introduce new variables $r_{i j}=\delta \rho_{i j}$ which are associated to the eigenvalue 0 of the Poisson tensor.

This scheme is general. When the Poisson tensor possesses vanishing eigenvalues (related to the existence of constants of the motion), it is unavoidable, in order to obtain a canonical parametrization, to introduce additional variables that Poisson-commute with the CCV. Let us illustrate this point by looking for a canonical parametrization of $\rho$ in the pure state case $\left(\beta^{-1}=0\right)$. Using (5.1), the matrix $\rho$ can be expressed in terms of the $(n \times n)$ matrices (see [17])

$$
\left\{\begin{aligned}
\Gamma_{i j} & =\left\langle\bar{x}_{i} \bar{x}_{j}\right\rangle \\
\Lambda_{i j} & =\left\langle\bar{p}_{i} \bar{p}_{j}\right\rangle \\
\Delta_{i j} & =\left\langle\bar{x}_{i} \bar{p}_{j}+\bar{p}_{j} \bar{x}_{i}\right\rangle
\end{aligned}\right.
$$

of which the evolution equations write

$$
\left\{\begin{array}{l}
\dot{\Gamma}=2\left(2 \frac{\partial \mathcal{F}}{\partial \Delta}+\Delta \frac{\partial \mathcal{F}}{\partial \Lambda}\right)_{s} \\
\dot{\Lambda}=-2\left(2 \Lambda \frac{\partial \mathcal{F}}{\partial \Delta}+\frac{\partial \mathcal{F}}{\partial \Gamma} \Delta\right)_{s} \\
\dot{\Delta}=-2\left[\Delta, \frac{\partial \mathcal{F}}{\partial \Delta}\right]-4\left(\Gamma \frac{\partial \mathcal{F}}{\partial \Gamma}-\frac{\partial \mathcal{F}}{\partial \Lambda} \Lambda\right)
\end{array}\right.
$$


where the index $s$ denotes the symmetric parts of the matrices involved. The pure state property $\rho(\rho+1)=0$ is equivalent to the conditions

$$
\left\{\begin{aligned}
I_{i j} & \equiv 4(\Gamma \Lambda)_{i j}-\left(\Delta^{2}\right)_{i j}=\delta_{i j} \\
(\Delta \Gamma)_{j i} & =(\Delta \Gamma)_{i j}, \\
(\Lambda \Delta)_{j i} & =(\Lambda \Delta)_{i j} .
\end{aligned}\right.
$$

As already mentioned in section 2, these conditions are preserved by the equations (5.9) if they were initially fulfilled. This is a typical example of vanishing eigenvalues of the Poisson tensor which depend on the initial conditions. Having chosen these initial conditions, one may in principle find a canonical form for the equations (5.9) with the additional constraints (5.10). But in our particular case, we can explicitly eliminate these constraints by defining the two symmetric matrices

$$
\left\{\begin{array}{l}
F=\Gamma, \\
G=\frac{1}{4} \Gamma^{-1} \Delta .
\end{array}\right.
$$

Indeed, the evolution of $F$ and $G$ is governed by Hamilton's equations

$$
\left\{\begin{array}{l}
\dot{F}=\frac{\partial \mathcal{E}}{\partial G}, \\
\dot{G}=-\frac{\partial \mathcal{E}}{\partial F},
\end{array}\right.
$$

and $\Lambda$ will be given by $\Lambda=\frac{1}{4} F^{-1}+4 G F G$. It should be noticed however, that this canonical parametrization is not global since it does not span the whole manifold $\rho$, but is rather valid on a sheet characterized by the special initial conditions.

Our last illustration in the search for CCV is the one-dimensional case. The matrices defined in (5.8) become c-numbers. One can see that the quantity $I$ is conserved, whatever the initial conditions. In particular, the initial 
state needs not be pure. In order to rewrite the TDHB equations, it is possible to keep the definitions (5.11). Equations (5.12) then remain valid (with $\Lambda$ replaced by $\left.I / 4 F+4 F G^{2}\right)$. But sometimes, it is preferable to work with variables that have the dimensionalities of $x$ and $p$. Hence, if we define

$$
\left\{\begin{array}{l}
w=\sqrt{\Gamma} \\
u=\frac{1}{2} \frac{\Delta}{\sqrt{\Gamma}}
\end{array}\right.
$$

we obtain from $(5.9)$

$$
\left\{\begin{array}{l}
\dot{w}=\frac{\partial \mathcal{F}}{\partial u}, \\
\dot{u}=-\frac{\partial \mathcal{F}}{\partial w} .
\end{array}\right.
$$

We arrive at the conclusion that a quantum one-dimensional bosonic system in the TDHB approximation evolves as a classical Hamiltonian system with two coupled degrees of freedom. In contrast with the $n$-dimensional case, this result holds for systems at non-zero temperature.

\section{Conclusions}

Upon selecting a trial time-dependent density operator $\mathcal{D}(t)$ belonging to the class of gaussian bosonic operators and a trial time-dependent observable $\mathcal{A}(t)$ belonging to the class of "one-body" operators, we derived, from the BV variational principle, the evolution equations (labeled by TDHB) for the first three contractions of $\mathcal{D}(t)$ namely, the "partition function", the expectation value $\langle\alpha\rangle$ of the "one-boson" operator $\alpha$ and the contraction matrix $\rho$.

We have investigated the relation of these TDHB equations with the hamitlonian formalism. We have exhibited the Lie-Poisson bracket associ- 
ated with the TDHB equations and with their linearized form (LTDHB) by exploiting the Lie algebra of the set $\{1, \alpha, \alpha \alpha\}$ of the relevant observables. We have also performed a stability analysis of TDHB and LTDHB.

The fact that the Poisson tensor associated with this bracket is nonsymplectic, brings forward the main difference between the TDHB and Hamiltonian evolutions, namely, that the former is non-canonical. This led us to wonder about the possible existence of canonically conjugate variables. The very existence of vanishing eigenvalues of the Poisson tensor, the dependence of the latter on the point of the manifold and, last but not least, the variation of the multiplicity of the zero eigenvalues from point to point, render the search for global sets of CCV highly non-trivial in the general case. Local canonical parametrizations are however possible in spite of the additional constraints that one must introduce to account for the conservation equations. This was shown to be the case for the LTDHB equations. We have also shown in this context, that the TDHB equations, in the pure state limit, can be cast in an Hamiltonian form by removing the constraints associated with the pure state conditions, and therefore reducing the number of degrees of freedom.

The study of the mathematical properties of the TDHB approximation may also prove useful in the search for consistent extensions. For instance, one could "re-quantize" the TDHB equations by suitably deforming their associated Lie-Poisson bracket.

We are indebted to R. Balian, M. Vénéroni, C. Martin, H. Flocard and D. Vautherin for fruitful discussions. 


\section{References}

[1] P. A. M. Dirac, Proc. Cambridge Philos. Soc. 26 (1930), 376.

[2] V. I. Arnold, "Mathematical Methods of Classical Mechanics", Graduate Texts in Mathematics, Vol. 60, Springer-Verlag, New York, (1978).

[3] J. E. Marsden (Ed), "Fluids and Plasmas: Geometry and Dynamics", Contemporary Mathematics, Vol. 28, Amer. Math. Soc., Providence, RI, 1984.

[4] R. Balian and M. Vénéroni, Ann. of Phys. (N.Y.) 195 (1989), 324 and references therein.

[5] J. E. Marsden, P. J. Morrison and A. Weinstein, "The Hamiltonian Structure of the BBGKY Hierarchy Equations", Cont. Math. 28 (1984), 115; J. E. Marsden and P. J. Morrison, "Noncanonical Hamiltonian Field Theory and Reduced MHD", Cont. Math. 28 (1984), 133.

[6] P. J. Morrison, "Poisson Brackets for Fluids and Plasmas", in Mathematical Methods in Hydrodynamics and Integrability in Related Dynamical Systems, AIP Conf. Proc., 88, La Jolla, (1982), M. Tabor (Ed).

[7] E. C. G. Sudarshan and N. Mukunda, "Classical Mechanics: A Modern Perspective", 2nd Edition, Krieger, (1983).

[8] R. G. Littlejohn, J. Math. Phys. 20 (1979), 2445; "Singular Poisson Tensors", from Mathematical Methods in Hydrodynamics and Integrability 
in Related Dynamical Systems, AIP Conf. Proc., 88, La Jolla, (1982), 47, M. Tabor (Ed).

[9] J. Frenkel, "Wave Mechanics, Advanced General Theory" Oxford Univ. Press (Clarendon), Oxford (1934); D. J. Thouless, "The Quantum Mechanics of Many-Body Systems", Academic Press, New York/London (1961); P. Ring and P. Schuck, "The Nuclear Many-Body Problem", Springer-Verlag, New York/Heidelberg/Berlin, (1980).

[10] A. L. Fetter and J. D. Walecka, "Quantum Theory of Many-Particle Systems", chap. 14, McGraw-Hill, N.Y. 1971.

[11] L. Landau and E. Lifshitz, "Physique Statistique", 2ème partie. Ed. Librairie du globe. France, 1978; P. Nozières and D. Pines, "The Theory of Quantum Liquids" vol.II. Addison-Wesley Publishing Company 1990.

[12] E. P. Gross, Nuovo Cimento 20 (1961), 454; L. Pitaevskii, Soviet Phys. JETP 13 (1961), 451.

[13] A. Griffin, D. W. Snoke and S. Stringari (Eds.) "Bose-Einstein Condensation", Cambridge Univ. Press, Cambridge, England, 1995.

[14] R. Balian and M. Vénéroni, Phys. Rev. Lett. 47 (1981), 1353, 1765(E).

[15] R. Balian and M. Vénéroni, Ann. of Phys. (N.Y.) 164 (1985), 334.

[16] R. Balian and M. Vénéroni, Ann. of Phys. (N.Y.) 187 (1988), 29.

[17] M. Benarous, Thesis. IPN-Orsay-France October 1991. 
[18] M. Benarous and H. Flocard, in preparation.

[19] R. Balian and E. Brezin, Nuovo Cimento, B64 (1969), 37.

[20] R. Balian and M. Vénéroni, Nucl. Phys. B408 (1993), 445.

[21] P. Kramer and M. Saraceno, "Geometry of the Time-Dependent Variational Principle", Lect. Notes in Phys. vol. 140 (1981), Springer-Verlag, Berlin.

[22] A. K. Kerman and S. E. Koonin, Ann. of Phys. (N.Y.) 100 (1976), 332.

[23] A. Weinstein, "Stability of Poisson-Hamilton Equilibria", Cont. Math. 28 (1984), 3; "The Local Structure of Poisson Manifolds", J. Diff. Geom. 18 (1983), 523. 